\title{
Gravitationally bound cores in a molecular cirrus cloud
}

\author{
A. Heithausen ${ }^{1}$, F. Bertoldi ${ }^{2}$, and F. Bensch ${ }^{3,4}$ \\ 1 Radioastronomisches Institut der Universität Bonn, Auf dem Hügel 71, 53121 Bonn, Germany \\ 2 Max-Planck-Institut für Radioastronomie, Auf dem Hügel 69, 53121 Bonn, Germany \\ 3 I. Physikalisches Institut der Universität zu Köln, Zülpicher Str. 77, 50937 Köln, Germany \\ 4 Harvard-Smithonian Center for Astrophysics, 60 Garden St., Cambridge, MA 02138, USA
}

Received 23 November 2001 / Accepted 18 December 2001

\begin{abstract}
Using MAMBO at the IRAM $30 \mathrm{~m}$ telescope we have observed a dense core in the cirrus cloud MCLD $123.5+24.9$ in the dust continuum emission at $250 \mathrm{GHz}$. The core is detected as an elongated filament with an extent of $4.2 \times 0 . ' 7$, corresponding to $0.18 \mathrm{pc} \times 0.03 \mathrm{pc}$ at an adopted distance of $150 \mathrm{pc}$. We find a close correlation between the continuum emission and previously observed $\mathrm{C}^{18} \mathrm{O}(1 \rightarrow 0)$ line emission. Using standard dust models we derive hydrogen column densities of up to $10^{22} \mathrm{~cm}^{-2}$. The total mass for the filament is about $0.66 M_{\odot}$.

We also present observations of the $\mathrm{HC}_{3} \mathrm{~N}(3 \rightarrow 2),(4 \rightarrow 3)$, and $(10 \rightarrow 9)$ emission lines obtained with the MPIfR $100 \mathrm{~m}$ and the IRAM $30 \mathrm{~m}$ telescopes. The distribution is very different from the dust continuum and the $\mathrm{C}^{18} \mathrm{O}(1 \rightarrow 0)$ line emission. $\mathrm{HC}_{3} \mathrm{~N}$ is concentrated in two distinct clumps located at the ends of the filament seen in the other tracers. Based on a $L V G$ analysis of the $\mathrm{HC}_{3} \mathrm{~N}$ transitions we derive column densities of $N\left(\mathrm{HC}_{3} \mathrm{~N}\right) / \Delta v \approx 10^{13} \mathrm{~cm}^{-2} / \mathrm{km} \mathrm{s}^{-1}$ and volume densities of $n\left(\mathrm{H}_{2}\right) \approx 10^{5} \mathrm{~cm}^{-3}$. We find that the $\mathrm{HC}_{3} \mathrm{~N}$ clumps have masses of 0.13 and $0.19 M_{\odot}$. Our data demonstrate that the cirrus cloud cores are gravitationally bound, and that they show chemical structure indicating different evolutionary stages within the cloud.
\end{abstract}

Key words. stars: formation - ISM: abundances - ISM: clouds - ISM: individual objects: MCLD 123.5+24.9 ISM: molecules - ISM: dust

\section{Introduction}

Stars form in dense cores of molecular clouds. One of the questions of current interest focuses on how dense cores form and how they eventually evolve into the protostellar Class 0 stage (e.g. Ward-Thompson et al. 1999). Most of the studies available concentrate on dense cores in regions with known star-forming activities (e.g. Motte et al. 1998). Galactic cirrus clouds have long been thought to be unable of forming any stars, because of their strong turbulent support against self-gravity (Magnani et al. 1987; Heithausen 1996). It did not surprise then that searches for T Tauri stars associated with high-latitude clouds remained unsuccessful (Martin \& Kun 1996; Hearty et al. 1999), except maybe L1457, where several T Tauri stars were found (Hearty et al. 2000), but for which an actual spatial coincidence between the stars and the cloud is not well established.

The discovery of $\mathrm{T}$ Tauri stars far from actively star forming regions (e.g., Neuhäuser 1999) raises the question

Send offprint requests to: A. Heithausen,

e-mail: heith@astro.uni-bonn.de of their origin. Are they runaway stars that were ejected from the parent molecular cloud, or were they born in small cloudlets (Feigelson 1996) which then dispersed? In this context the star-forming abilities of molecular cirrus clouds, which are mostly found at high galactic latitudes, is of great interest. Recently, CS observations of the cirrus cloud MCLD 123.5+24.9 (Heithausen 1999) showed spectroscopic signatures of infall motion (cf. Myers et al. 1996; Mardones et al. 1997) in one of its three CS cores, suggesting that star-formation is indeed possible in this type of clouds.

MCLD $123.5+24.9$ is a dense molecular cloud located in the Polaris Flare, a large molecular cirrus cloud in the direction of the north celestial pole (Heithausen \& Thaddeus 1990). Its distance is between $130 \mathrm{pc}$ and $240 \mathrm{pc}$ (cf. Heithausen et al. 1993), and we shall here adopt a value of $150 \mathrm{pc}$. The first molecules detected towards MCLD $123.5+24.9$ were $\mathrm{CO},{ }^{13} \mathrm{CO}, \mathrm{H}_{2} \mathrm{CO}$, and $\mathrm{OH}$ (Großmann et al. 1990), which shows an enhanced abundance. Later observations found $\mathrm{NH}_{3}, \mathrm{HCO}^{+}, \mathrm{HCN}$, and HNC, with abundances typical of dark clouds (Großmann \& Heithausen 1992), and implied kinetic temperatures 
Table 1. Parameters for the $\mathrm{HC}_{3} \mathrm{~N}$ observations.

\begin{tabular}{llllllll}
\hline Molecule & transition & $\begin{array}{l}\text { Frequency } \\
(\mathrm{GHz})\end{array}$ & Telescope & $\begin{array}{l}F W H M \\
\left({ }^{\prime \prime}\right)\end{array}$ & $\begin{array}{l}\eta_{\mathrm{mb}} \\
\left(\mathrm{km} \mathrm{s}^{-1}\right)\end{array}$ & Positions \\
\hline $\mathrm{HC}_{3} \mathrm{~N}$ & $J=3 \rightarrow 2 ; F=3 \rightarrow 2$ & 27.294295 & MPIfR $100 \mathrm{~m}$ & 32 & 0.42 & 0.054 & 52 \\
$\mathrm{HC}_{3} \mathrm{~N}$ & $J=4 \rightarrow 3 ; F=4 \rightarrow 3$ & 36.392332 & MPIfR 100 m & 26 & 0.42 & 0.060 & 2 \\
$\mathrm{HC}_{3} \mathrm{~N}$ & $J=10 \rightarrow 9$ & 90.978993 & IRAM 30 m & 27 & 0.81 & 0.064 & 82 \\
\hline
\end{tabular}

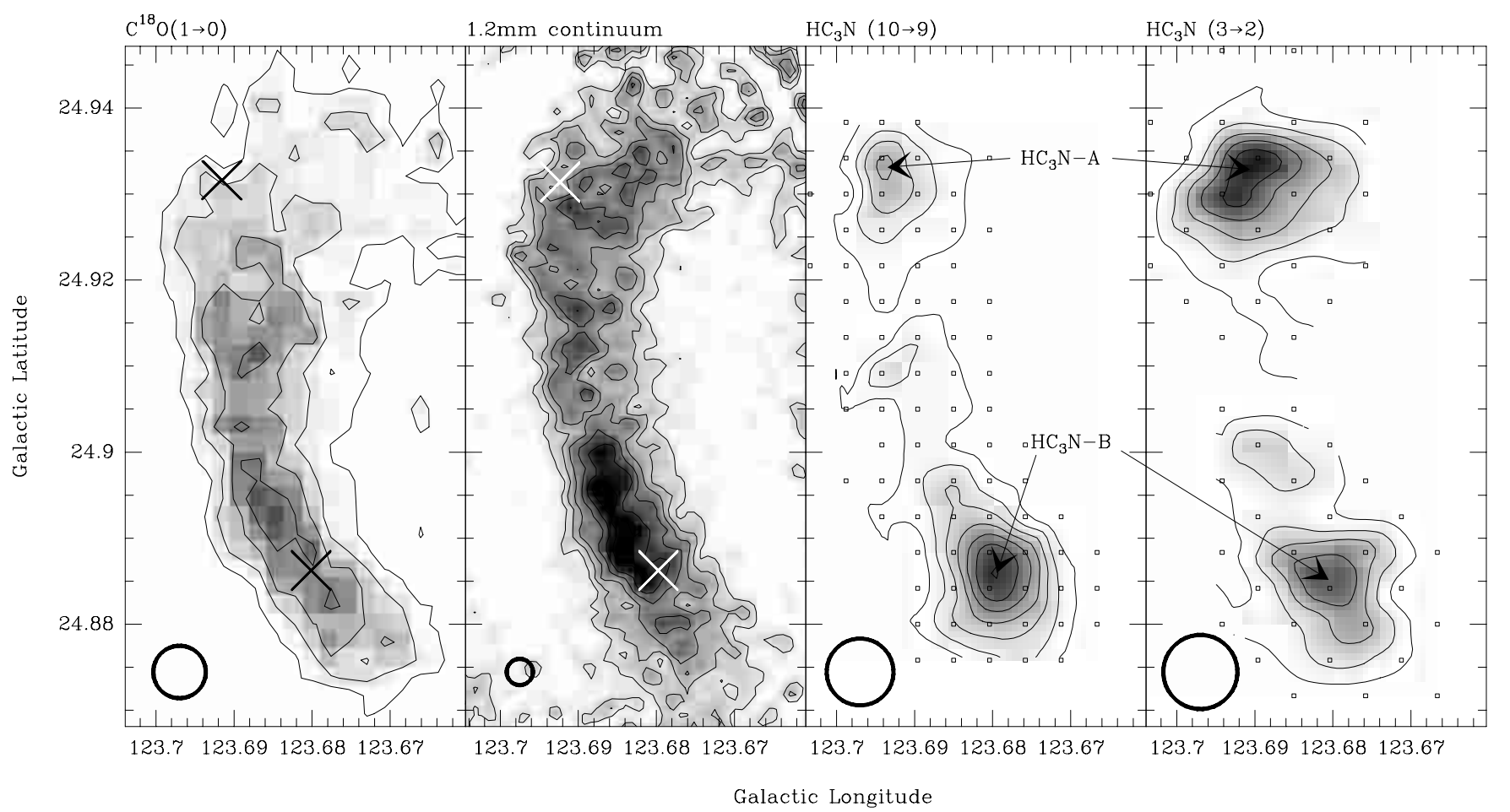

Fig. 1. Comparison of the distribution of $\mathrm{C}^{18} \mathrm{O}$ (taken from Falgarone et al. 1998), dust continuum and $\mathrm{HC}_{3} \mathrm{~N}$. Contours are every $0.25 \mathrm{~K} \mathrm{~km} \mathrm{~s}^{-1}$ starting at $0.5 \mathrm{~K} \mathrm{~km} \mathrm{~s}^{-1}$ for the $\mathrm{C}^{18} \mathrm{O}(1 \rightarrow 0)$ line, every $2 \mathrm{mJy} /$ beam starting at $0 \mathrm{mJy} /$ beam for the dust continuum map, every $0.05 \mathrm{~K} \mathrm{~km} \mathrm{~s}^{-1}$ starting at $0.05 \mathrm{~K} \mathrm{~km} \mathrm{~s}^{-1}$ for the $\mathrm{HC}_{3} \mathrm{~N}(10 \rightarrow 9)$ line, and every $0.1 \mathrm{~K} \mathrm{~km} \mathrm{~s}^{-1}$ starting at $0.1 \mathrm{~K} \mathrm{~km} \mathrm{~s}^{-1}$ for the $\mathrm{HC}_{3} \mathrm{~N}(3 \rightarrow 2)$ line. Beam sizes are indicated in the lower left corner of the maps. Observed $\mathrm{HC}_{3} \mathrm{~N}$ positions are marked in the respective maps. The arrows point towards the centres of $\mathrm{HC}_{3} \mathrm{~N}-\mathrm{A}$ and $\mathrm{B}$, which are also the positions, for which the $\mathrm{HC}_{3} \mathrm{~N}(4 \rightarrow 3)$ spectra have been obtained. The "X"s in the left two maps mark the centres of $\mathrm{HC}_{3} \mathrm{~N}-\mathrm{A}$ and $\mathrm{B}$.

between $6 \mathrm{~K}$ and $15 \mathrm{~K}$. Further molecules detected here are $\mathrm{C}_{3} \mathrm{H}_{2}$, SO, CS, CCH (Gerin et al. 1997; Heithausen et al. 1995, 1999). Dust continuum observations made by IRAS, ISO, and PRONAOS (a balloon-borne submmphotometer) show steep spectrum emission from cold $\left(T_{\mathrm{d}}=13 \mathrm{~K}\right)$ dust (Bernard et al. 1999).

Here we present $1.2 \mathrm{~mm}(250 \mathrm{GHz})$ radio continuum observations of MCLD 123.5+24.9 obtained with the 37-channel Max-Planck Millimeter Bolometer (MAMBO) array at the IRAM $30 \mathrm{~m}$ telescope. We also show observations of three $\mathrm{HC}_{3} \mathrm{~N}$ transitions obtained with the MPIfR $100 \mathrm{~m}$ and the IRAM $30 \mathrm{~m}$ telescopes. Our data demonstrate that the cirrus cloud cores are gravitationally bound, and that they show chemical structure indicating different evolutionary stages within the cloud.

\section{Observations}

Table 1 summarizes the basic parameters of the spectroscopic observations. The $\mathrm{HC}_{3} \mathrm{~N}(3 \rightarrow 2)$ and $(4 \rightarrow 3)$ spectra were obtained in June 2000 with the MPIfR $100 \mathrm{~m}$ radio telescope. For the lower transition we obtained a complete map with 52 positions. In the direction of the most intense $(3 \rightarrow 2)$ line emission we also obtained two spectra in the $(4 \rightarrow 3)$ transition. The velocity resolution of the autocorrelation spectrometer was $0.06 \mathrm{~km} \mathrm{~s}^{-1}$. The rms noise level in the final spectra is below $0.16 \mathrm{~K}$ for the $(3 \rightarrow 2)$, and $0.26 \mathrm{~K}$ for the $(4 \rightarrow 3)$ data. The $\mathrm{HC}_{3} \mathrm{~N}(10 \rightarrow 9)$ transition was observed with the IRAM $30 \mathrm{~m}$ telescope in September 2000 over a full map with 82 positions on a $15^{\prime \prime}$ rectangular grid, with an rms noise in the final spectra below $0.05 \mathrm{~K}$.

Millimeter continuum observations were made on four different dates in March 2000 with the 37-channel 
bolometer MAMBO at the IRAM $30 \mathrm{~m}$ telescope. MAMBO is sensitive between about 210 and $290 \mathrm{GHz}$, with an effective frequency of $250 \mathrm{GHz}$ for steep thermal spectra. Eight maps covering about $4^{\prime} \times 4^{\prime}$ each were coadded for the final map. The observations were taken in double-beam on-the-fly mode, i.e., chopping the secondary mirror in azimuth by $50^{\prime \prime}$ to $70^{\prime \prime}$ at $2 \mathrm{~Hz}$, and scanning the sky in azimuth at a speed of 4 or $5^{\prime \prime} \mathrm{s}^{-1}$, then moving in elevation by $4^{\prime \prime}$. The total observing time was about 8 hours. The maps were taken under variable winter weather conditions, with line of sight opacities between 0.2 and 0.7 . The effective beam $F W H M$ is $\approx 11^{\prime \prime}$.

To our knowledge this is the first millimeter bolometer map of a galactic cirrus core. In Fig. 1 we compare the spectroscopic and continuum data to a complete $\mathrm{C}^{18} \mathrm{O}(1 \rightarrow 0)$ map of that cloud, which was previously obtained by Falgarone et al. (1998).

\section{Dust continuum}

Broadband continuum emission at $\mathrm{mm}$ and submm wavelengths yields information on the distribution of dust in molecular clouds. The dust emission provides an independent measure for the total mass surface density, which is otherwise hard to estimate, especially in high-latitude clouds.

As seen in Fig. 1, the core is clearly detected as an elongated filament with an extent of $4.2 \times 0 . ' 7$, corresponding to $0.18 \mathrm{pc} \times 0.03 \mathrm{pc}$ at the adopted distance of $150 \mathrm{pc}$. The flux density, $S_{\nu}$, in a Gaussian beam with a halfpower beamwidth $\Theta$, of thermal emission by dust with a temperature $T_{\mathrm{d}}$ and an optical depth $\tau_{\lambda}$ is

$S_{\nu}=\frac{\pi}{4 \ln 2} \Theta^{2}\left(1-\mathrm{e}^{-\tau_{\lambda}}\right) B_{\nu}\left(T_{\mathrm{d}}\right)$.

Following Mezger et al. (1995), the dust optical depth through a hydrogen nuclei column density $N_{\mathrm{H}}$ is

$\tau_{\lambda}=b C\left(\frac{\lambda}{\mathrm{mm}}\right)^{m}\left(\frac{Z}{Z_{0}}\right) N_{\mathrm{H}}$,

where $\frac{Z}{Z_{0}}$ is the gas metallicity relative to the solar value, where $C=7 \times 10^{-27} \mathrm{~cm}^{2}$ is the Draine \& Lee (1984) dust absorption cross section per $\mathrm{H}$ nucleon at wavelength $1 \mathrm{~mm}$, and $b$ is an empirical correction factor accounting for differences to the Draine \& Lee diffuse cloud grains; $b \approx 1-2$ for grains in dark clouds, and we adopt $b=1.9$ (Mezger et al. 1995). For $m=2$ and $T_{\mathrm{d}}=13 \mathrm{~K}$ (Bernard et al. 1999), in the case of $\tau_{\lambda} \ll 1$ we find a linear relation between the $\mathrm{H}$ column density and the dust continuum emission:

$N\left(\mathrm{H}_{2}\right) \approx N_{\mathrm{H}} / 2=1.1 \times 10^{21}\left(\frac{S_{\nu}}{\mathrm{mJy} \mathrm{beam}^{-1}}\right) \mathrm{cm}^{-2}$,

assuming solar metallicity. Thus the mean $250 \mathrm{GHz}$ flux density of $5.2 \mathrm{mJy}^{\text {beam }}{ }^{-1}$ averaged over the whole filament corresponds to a column $N\left(\mathrm{H}_{2}\right)=5.7 \times 10^{21} \mathrm{~cm}^{-2}$, and the peak flux density of $10.6 \mathrm{mJy} \mathrm{beam}^{-1}$ yields $N\left(\mathrm{H}_{2}\right)=11.7 \times 10^{21} \mathrm{~cm}^{-2}$. Assuming the core to be a

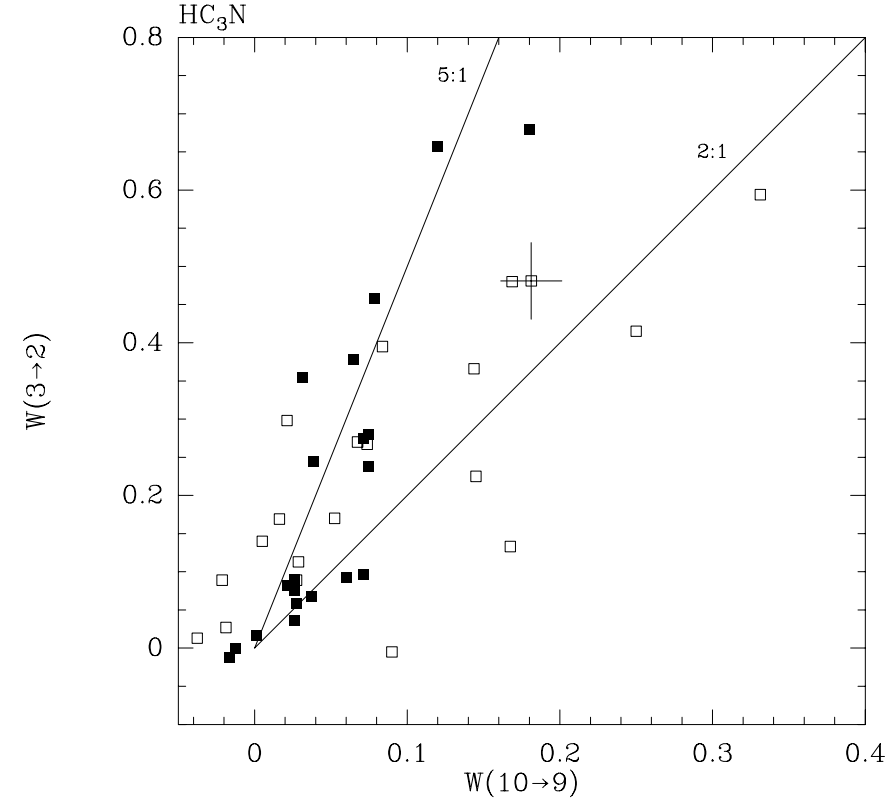

Fig. 2. Correlation of the velocity-integrated line intensities of the $\mathrm{HC}_{3} \mathrm{~N}(3 \rightarrow 2)$ and $(10 \rightarrow 9)$ transitions (in $\left.\mathrm{K} \mathrm{km} \mathrm{s}^{-1}\right)$. The values for $\mathrm{HC}_{3} \mathrm{~N}-\mathrm{A}$ are coded as filled squares, those of $\mathrm{HC}_{3} \mathrm{~N}-\mathrm{B}$ as open squares. The lines drawn are eye-fits to the data. The error bar represents a typical value for all the data.

cylindrical filament and not an edge-on sheet, we derive an average volume density $n\left(\mathrm{H}_{2}\right)=6.2 \times 10^{4} \mathrm{~cm}^{-2}$. The total mass of the filament (corrected for the contribution of helium) is $0.66 M_{\odot}$.

Figure 1 illustrates the good correspondence between the dust continuum emission and the integrated $\mathrm{C}^{18} \mathrm{O}$ line flux over most of the filament. Adopting an excitation temperature of $T_{\mathrm{ex}}=10 \mathrm{~K}$ the $\mathrm{C}^{18} \mathrm{O}$ emission peak flux yields a column density $N\left(\mathrm{C}^{18} \mathrm{O}\right)=1.2 \times 10^{15} \mathrm{~cm}^{-2}$. With the previously derived $\mathrm{H}_{2}$ column density, we find an abundance $\left[\mathrm{C}^{18} \mathrm{O}\right] /\left[\mathrm{H}_{2}\right] \approx 1 \times 10^{-7}$, which is in good agreement with the value previously found by Großmann \& Heithausen (1992) in this cloud.

\section{Cyanoacetylene}

Because of its low rotational constant cyanoacetylene $\left(\mathrm{HC}_{3} \mathrm{~N}\right)$ has many rotational lines which are easily accessible with ground-based radio telescopes. Therefore this molecule has widely been used to determine the excitation conditions in molecular clouds cores. We will use our $\mathrm{HC}_{3} \mathrm{~N}$ observations to derive the gas density in MCLD 123.5+24.9.

Figure 1 compares the intensity distributions of the dust continuum, and the $\mathrm{C}^{18} \mathrm{O}, \mathrm{HC}_{3} \mathrm{~N}(10 \rightarrow 9)$ and $(3 \rightarrow 2)$ line emission. For the $\mathrm{HC}_{3} \mathrm{~N}(3 \rightarrow 2)$ map the fluxes of the $F=2 \rightarrow 1,3 \rightarrow 2$ and $4 \rightarrow 3$ hyperfine components were coadded. The $\mathrm{HC}_{3} \mathrm{~N}$ emission looks very different from that of dust and $\mathrm{C}^{18} \mathrm{O} . \mathrm{HC}_{3} \mathrm{~N}$ is concentrated in two distinct clumps located at the ends of the filament traced by $\mathrm{CO}$ and dust. We shall denote the clump at higher galactic latitudes as $\mathrm{HC}_{3} \mathrm{~N}-\mathrm{A}$, the other one as $\mathrm{HC}_{3} \mathrm{~N}-\mathrm{B}$. 


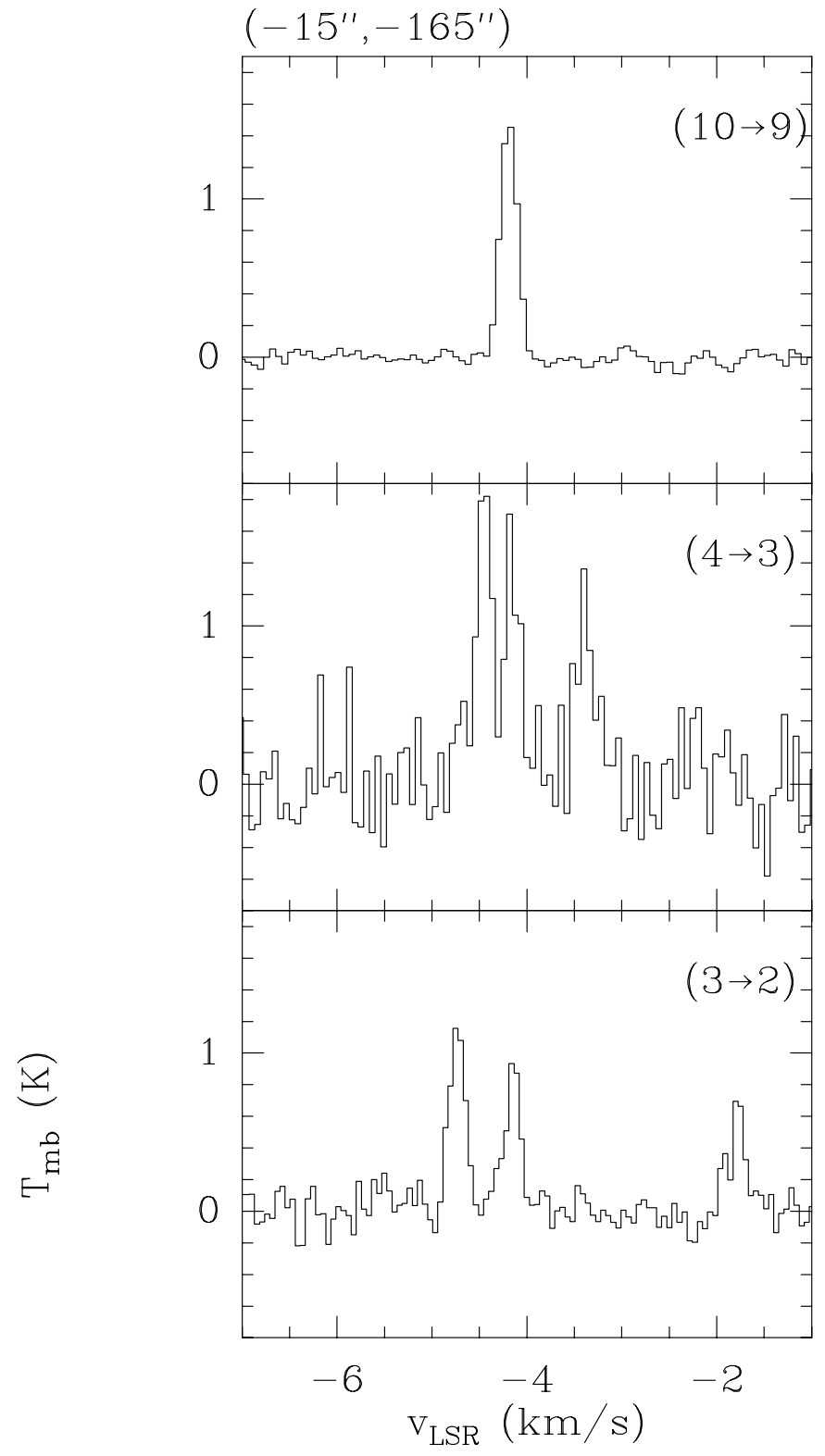

Fig. 3. Spectra of the $\mathrm{HC}_{3} \mathrm{~N}(J=3 \rightarrow 2),(4 \rightarrow 3)$ and $(10 \rightarrow 9)$ transitions towards the centre of $\mathrm{HC}_{3} \mathrm{~N}-\mathrm{B}$. In the $(3 \rightarrow 2)$ spectrum three hyperfine components are visible: the $F=(3 \rightarrow 2)$ component at $v_{\mathrm{LSR}}=-4.19 \mathrm{~km} \mathrm{~s}^{-1}$, the $(2 \rightarrow 1)$ component to the right and the $4 \rightarrow 3$ to the left. The $J=(4 \rightarrow 3)$ transition also shows three hyperfine components: the $F=4 \rightarrow 3$ component at $v_{\mathrm{LSR}}=-4.19 \mathrm{~km} \mathrm{~s}^{-1}$, the $3 \rightarrow 2$ component to the right and and the $5 \rightarrow 4$ to the left.

The positions and sizes of the two $\mathrm{C}^{18} \mathrm{O}$ clumps were determined through a 3-dimensional Gaussian fit (using GAUSSCLUMPS by Stutzki \& Güsten 1989) to each hyperfine component. The two clumps are well represented by the Gaussian intensity distribution, suggesting that they are centrally condensed. In Table 2 we list the average properties of the two clumps.

Figures 1 and 4 show that in between both clumps both transitions show only weak emission, lower by a factor 3 to 5 from the flux seen towards the clumps (see Table 3).

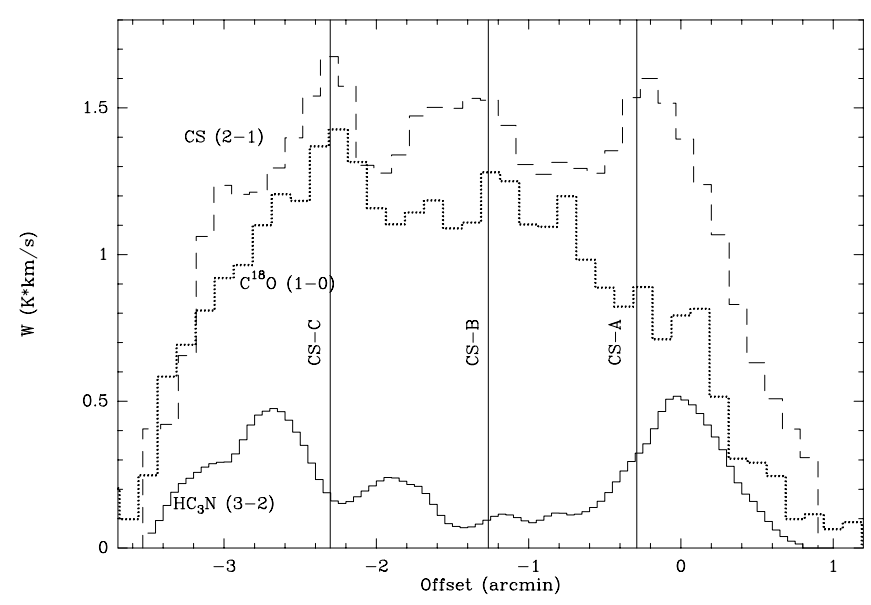

Fig. 4. Cut along a line connecting $\mathrm{HC}_{3} \mathrm{~N}-\mathrm{A}$ and $\mathrm{B}$ in the integrated $\mathrm{HC}_{3} \mathrm{~N}(3 \rightarrow 2)$ (solid line), the $\mathrm{C}^{18} \mathrm{O}(1 \rightarrow 0)$ (dotted line), and the CS $(2 \rightarrow 1)$ (dashed line) transitions. Offsets are relative to $\mathrm{HC}_{3} \mathrm{~N}-\mathrm{A}$. The location of the centres of the clumps CS-A, CS-B, and CS-C (Heithausen 1999) are marked by vertical lines.

In Fig. 2 we show a direct comparison of the $\mathrm{HC}_{3} \mathrm{~N}$ $(3 \rightarrow 2)$ and $(10 \rightarrow 9)$ integrated intensities. The ratio of the line temperatures is clearly different in the two clumps. An eye-fit to the data points yields approximately $R_{10 / 3} \equiv$ $W(10 \rightarrow 9) / W(3 \rightarrow 2)=1 / 5$ in $\mathrm{HC}_{3} \mathrm{~N}-\mathrm{A}$, and $R_{10 / 3}=$ $1 / 2$ in $\mathrm{HC}_{3} \mathrm{~N}-\mathrm{B}$.

Toward the peak intensity positions of the two clumps we also observed the $\mathrm{HC}_{3} \mathrm{~N}(4 \rightarrow 3)$ transition, and for $\mathrm{HC}_{3} \mathrm{~N}-\mathrm{B}$ we show this spectrum in Fig. 3, and fluxes are listed in Table 3. The three main hyperfine components are clearly detected toward both clumps. The expected ratio is for the components is $(F=5 \rightarrow 4):(F=4 \rightarrow$ $3):(F=3 \rightarrow 2)=1.00: 0.77: 0.58$ (Lafferty \& Lovas 1978), the observed ratio is $1.0:(0.8 \pm 0.1):(0.6 \pm 0.1)$, i.e. consistent with optically thin emission.

We have computed a large velocity gradient model to match the observed line intensities of the three transitions. Because the kinetic temperature as derived from ammonia observations (Großmann \& Heithausen 1992) is below $15 \mathrm{~K}$ we discuss the results for kinetic temperatures of 10 and $15 \mathrm{~K}$. The range of physical parameters reflects the $1 \sigma$ uncertainties of the observed intensities. For $T_{\text {kin }}=10 \mathrm{~K}$ we find $n_{\mathrm{H}_{2}} \approx(1.1 \pm 0.5) \times 10^{5} \mathrm{~cm}^{-3}$ toward $\mathrm{HC}_{3} \mathrm{~N}-\mathrm{A}$, and a factor 2.5 higher density for $\mathrm{HC}_{3} \mathrm{~N}-\mathrm{B}$. The peak column density $N\left(\mathrm{HC}_{3} \mathrm{~N}\right) / \Delta v \approx(1.6 \pm 0.8) \times 10^{13} \mathrm{~cm}^{-2} / \mathrm{km} \mathrm{s}^{-1}$ is similar in both clumps. For $T_{\text {kin }}=15 \mathrm{~K}$ the densities decreases by a factor of 2 but the column density is insensitive to the kinetic temperature.

\section{Discussion}

\subsection{Chemistry}

It had been noted previously (Heithausen et al. 1999) that the dense core in MCLD 123.5+24.9 shows a different structure when traced by different molecular emission lines. The most striking difference apparent from our data 
Table 2. Properties of the $\mathrm{HC}_{3} \mathrm{~N}$ clumps.

\begin{tabular}{|c|c|c|c|c|c|c|c|c|c|c|}
\hline Core & $\begin{array}{l}l \\
{[\mathrm{deg}]}\end{array}$ & $\begin{array}{l}b \\
{[\mathrm{deg}]}\end{array}$ & $\begin{array}{l}v_{\mathrm{LSR}} \\
{\left[\mathrm{km} \mathrm{s}^{-1}\right]}\end{array}$ & $\begin{array}{l}\Delta v \\
{\left[\mathrm{kms}^{-1}\right]}\end{array}$ & $\begin{array}{l}\Delta l \times \Delta b \\
{[\operatorname{arcsec}]}\end{array}$ & $\begin{array}{l}r \\
{[\mathrm{pc}]}\end{array}$ & $\begin{array}{l}n\left(\mathrm{H}_{2}\right) \\
{\left[10^{5} \mathrm{~cm}^{-3}\right]}\end{array}$ & $\begin{array}{l}\frac{N\left(\mathrm{HC}_{3} \mathrm{~N}\right)}{\Delta v} \\
{\left[10^{13} \frac{\mathrm{cm}^{-2}}{\mathrm{~km} \mathrm{~s}^{-1}}\right]}\end{array}$ & $\begin{array}{l}M_{\mathrm{cl}} \\
{\left[M_{\odot}\right]}\end{array}$ & $\begin{array}{l}M_{\text {vir }} \\
{\left[M_{\odot}\right]}\end{array}$ \\
\hline $\mathrm{HC}_{3} \mathrm{~N}-\mathrm{A}$ & 123.691 & 24.931 & -4.59 & 0.19 & $35 \times 33$ & 0.025 & $0.6-1.6$ & $1.6 \pm 0.8$ & 0.13 & 0.19 \\
\hline $\mathrm{HC}_{3} \mathrm{~N}-\mathrm{B}$ & 123.680 & 24.886 & -4.19 & 0.19 & $20 \times 42$ & 0.021 & $1.6-4.0$ & $1.6 \pm 0.8$ & 0.19 & 0.16 \\
\hline
\end{tabular}

Remarks: values are averages of the three hyperfine components. $\Delta v, \Delta l$, and $\Delta b$ are deconvolved from the instrumental resolution.

Table 3. $\mathrm{HC}_{3} \mathrm{~N}$ line fluxes (summed over the finestructure components) and their ratios.

\begin{tabular}{llllllll}
\hline Region & $\langle W(3 \rightarrow 2)\rangle$ & $W(4 \rightarrow 3)^{a}$ & $\langle W(10 \rightarrow 9)\rangle$ & $\left\langle\frac{W(10 \rightarrow 9)}{W(4 \rightarrow 3)}\right\rangle$ & $\left\langle\frac{W(3 \rightarrow 2)}{W\left(\mathrm{C}^{18} \mathrm{O} 1 \rightarrow 0\right)}\right\rangle$ & $\left\langle\frac{W(10 \rightarrow 9)}{W\left(\mathrm{C}^{18} \mathrm{O} \rightarrow 0\right)}\right\rangle$ & Identification \\
& {$\left[\mathrm{K} \mathrm{km} \mathrm{s}^{-1}\right]$} & {$\left[\mathrm{K} \mathrm{km} \mathrm{s}^{-1}\right]$} & {$\left[\mathrm{K} \mathrm{km} \mathrm{s}^{-1}\right]$} & & & \\
\hline $\mathrm{HC}_{3} \mathrm{~N}-\mathrm{A}$ & $0.61 \pm 0.02$ & $0.83 \pm 0.10$ & $0.170 \pm 0.010$ & 0.2 & $0.69 \pm 0.05$ & $0.20 \pm 0.02$ & $\mathrm{NH}_{3}-\mathrm{A}^{b}, \mathrm{CS}^{-\mathrm{A}^{c}}$ \\
$\mathrm{HC}_{3} \mathrm{~N}-\mathrm{B}$ & $0.57 \pm 0.02$ & $0.90 \pm 0.06$ & $0.333 \pm 0.006$ & 0.5 & $0.39 \pm 0.05$ & $0.23 \pm 0.02$ & $\mathrm{NH}_{3}-\mathrm{B}^{b},{\mathrm{CS}-\mathrm{C}^{c}}^{c}$ \\
Intercore & $0.12 \pm 0.02$ & - & $0.060 \pm 0.004$ & - & $0.10 \pm 0.03$ & $0.06 \pm 0.01$ & ${\mathrm{CS}-\mathrm{B}^{c}}$ \\
\hline
\end{tabular}

Remarks: ${ }^{a}$ : single position; ${ }^{b}$ : Großmann \& Heithausen (1992); ${ }^{c}$ : Heithausen (1999).

is that $\mathrm{C}^{18} \mathrm{O}$ and the dust continuum emission trace a narrow filament, whereas the $\mathrm{HC}_{3} \mathrm{~N}$ emission is concentrated in two clumps, $\mathrm{HC}_{3} \mathrm{~N}-\mathrm{A}$ and $\mathrm{B}$, located on both ends of the filament. These clumps coincide with two ammonia emission clumps, $\mathrm{NH}_{3}-\mathrm{A}$ and $\mathrm{B}$, detected by Großmann \& Heithausen (1992). Although the $\mathrm{NH}_{3}(1,1)$ inversion transition was observed with lower $\left(40^{\prime \prime}\right)$ angular resolution, there appears also an emission gap between the $\mathrm{NH}_{3}$ clumps, just as we found for $\mathrm{HC}_{3} \mathrm{~N}$.

The cross cut shown in Fig. 4 shows that the $\mathrm{HC}_{3} \mathrm{~N}$ clumps somewhat overlap with the CS clumps A and C, but their peak intensity is clearly shifted towards the ends of the CS and $\mathrm{C}^{18} \mathrm{O}$ filament. The CS clump B has no corresponding $\mathrm{HC}_{3} \mathrm{~N}$ feature.

For a quantitative comparison between the $\mathrm{HC}_{3} \mathrm{~N}$ and $\mathrm{C}^{18} \mathrm{O}$ emission we computed average intensities for both species toward the $\mathrm{HC}_{3} \mathrm{~N}$ clumps and in the area in between. The ratios listed in Table 3 show that the relative intensity drops by a factor 3 to 7 in between the $\mathrm{HC}_{3} \mathrm{~N}$ clumps.

The strong drop in $\mathrm{HC}_{3} \mathrm{~N}$ intensity relative to $\mathrm{C}^{18} \mathrm{O}$ may be caused by a change in excitation or abundance. It is unlikely that the excitation conditions vary along the filament, since its gas column density, as traced by the $\mathrm{C}^{18} \mathrm{O}$ and dust emission, rather increases toward the middle of the filament. A near constant gas density is also implied by the CS $(5 \rightarrow 4)$ observations (Heithausen 1999, and new unpublished observations). A drop in the kinetic temperature also seems unlikely, since this would also affect the $\mathrm{C}^{18} \mathrm{O}$ and dust emission.

Could it therefore be that the relative intensity gradients are due to a lower $\mathrm{HC}_{3} \mathrm{~N}$ and $\mathrm{NH}_{3}$ abundance compared with $\mathrm{C}^{18} \mathrm{O}$ and $\mathrm{CS}$ in the middle of the filament? The chemistry of $\mathrm{HC}_{3} \mathrm{~N}$ has been studied in great detailed, finding that it is an early-time molecule which has its highest abundance after $(0.3-1) \times 10^{6}$ years after the formation of the cloud (e.g. Nejad \& Wagenblast 1999; Gwenlan et al. $2000)$; over the next $(0.5-1.0) \times 10^{6}$ years the $\mathrm{HC}_{3} \mathrm{~N}$ abundance decreases by several orders of magnitudes. CS is the fastest-formed molecule, followed by $\mathrm{HC}_{3} \mathrm{~N}$, and then by $\mathrm{NH}_{3}$, which is formed at times later than $10^{6}$ years (Taylor et al. 1998). If we were to interpret the relative abundance gradients as an age effect, the clumps $\mathrm{HC}_{3} \mathrm{~N}-\mathrm{A}\left(=\mathrm{NH}_{3}-\mathrm{A}\right.$ and $\mathrm{CS}-\mathrm{A})$ and $\mathrm{HC}_{3} \mathrm{~N}-\mathrm{B}\left(=\mathrm{NH}_{3}-\mathrm{B}\right.$ and $\left.\mathrm{CS}-\mathrm{C}\right)$ would be the oldest, whereas CS-B must be the younger because it shows no ammonia emission and little cyanoacetylene.

\subsection{Stability}

The mass of the two $\mathrm{HC}_{3} \mathrm{~N}$ clumps may be estimated from the peak volume density derived from our excitation analysis (Sect. 4, Table 2), and, in agreement with the parameters derived from Gaussclumps listed in Table 2, assuming a Gaussian density profile with a $F W H M$, $r$, which we take as the geometric mean of the minor and major axis of the Gaussian intensity distribution. We then find $\mathrm{HC}_{3} \mathrm{~N}-\mathrm{A}$ to contain $0.13 M_{\odot}$, whereas $\mathrm{HC}_{3} \mathrm{~N}-\mathrm{B}$ has $0.19 M_{\odot}$. These masses are similar to those derived from the column density estimate of our bolometer map integrated over the extent of the $\mathrm{HC}_{3} \mathrm{~N}$ clumps.

To assess the clumps' stability we first analyse the $\mathrm{HC}_{3} \mathrm{~N}(10 \rightarrow 9)$ line widths. In Fig. 5 for each position we plot the line width against its peak brightness temperature. Because the clumps are centrally concentrated (s. Gaussian analysis above), the peak brightness temperature is also a measure of the distance from the cloud centre. For $\mathrm{HC}_{3} \mathrm{~N}-\mathrm{B}$ we find a trend for the spectra closer 


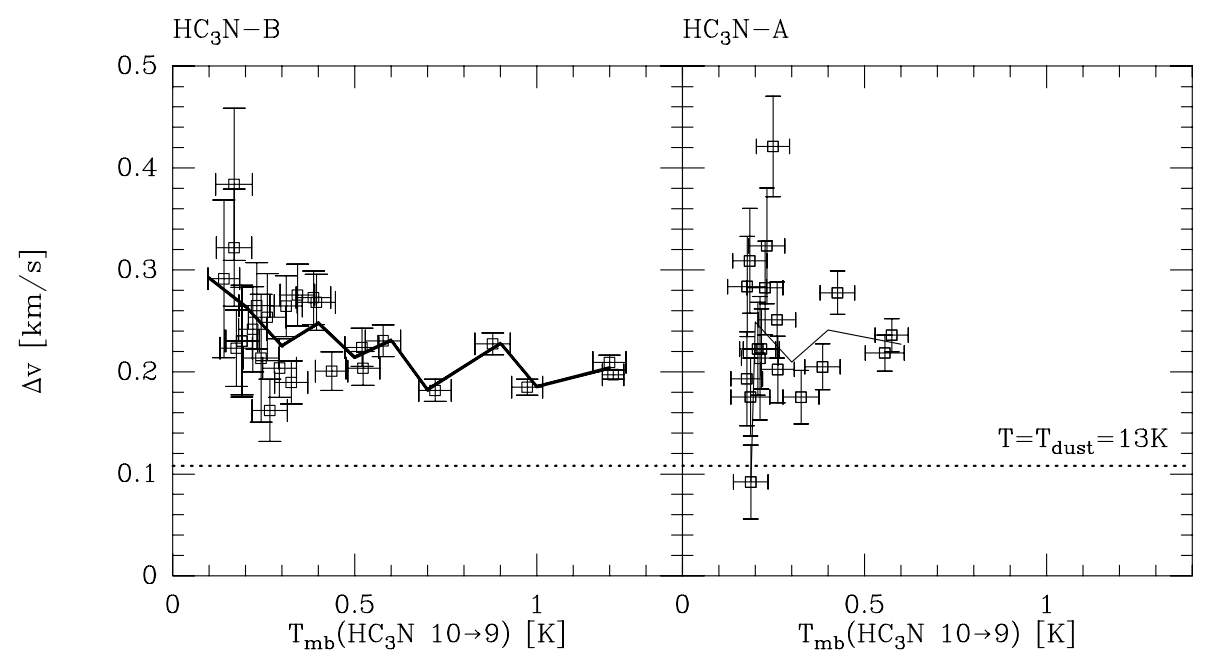

Fig. 5. Correlation of the line width $(F W H M)$ and the peak line temperature of the $\mathrm{HC}_{3} \mathrm{~N}(10 \rightarrow 9)$ line as derived from a Gaussian fit to the spectra for $\mathrm{HC}_{3} \mathrm{~N}-\mathrm{A}$ (right box) and $\mathrm{B}$ (left box). The solid line represents a running average for the values binned over $\Delta T=0.1 \mathrm{~K}$. The dotted line marks the thermal line width for a gas temperature $T_{\text {gas }}=T_{\text {dust }}=13 \mathrm{~K}$. Errorbars are $\pm 1 \sigma$.

to the clump center to show smaller line widths, but for $\mathrm{HC}_{3} \mathrm{~N}-\mathrm{A}$ no such correlation appears.

The clump mass may now be compared with the virial mass,

$M_{\mathrm{vir}}=5 \sigma^{2} r / G$

(Bertoldi \& McKee 1992), where $\sigma=\Delta v / 2.355$ is the one-dimensional gas velocity dispersion and $G$ is the gravitational constant. With the values listed in Table 2, the virial masses turn out to be very close to the masses we computed from the line and continuum emission, indicating that the clumps are gravitationally bound.

\section{Conclusions}

We have imaged the dust emission and $\mathrm{HC}_{3} \mathrm{~N}$ line emission in the cirrus cloud MCLD $123.5+24.9$ and find that most of the $\mathrm{HC}_{3} \mathrm{~N}$ emission arises from two condensations at the ends of an elongated filament which is traced by the dust continuum and by $\mathrm{C}^{18} \mathrm{O}$ and $\mathrm{CS}$ line emission. The $\mathrm{HC}_{3} \mathrm{~N}$ emission does correlate with previously reported $\mathrm{NH}_{3}$ emission, but does not correlate with the other tracers, suggesting either excitation or relative abundance gradients. We suggest that abundance gradients are likely to be responsible for the appearance, and that the abundance differences are due to chemical evolution. This would suggest that the $\mathrm{HC}_{3} \mathrm{~N}$ clumps are older than the center of the filament, which would be less than $3 \times 10^{5}$ years old and there has been no time for either the $\mathrm{HC}_{3} \mathrm{~N}$ or the $\mathrm{NH}_{3}$ to achieve a large abundance. Based on our $\mathrm{HC}_{3} \mathrm{~N}$ observations we find that the older clumps are gravitationally bound, and as signs for inward motion in one of them show (Heithausen 1999), they might be near gravitational collapse.

The detection of graviationally bound structures in cirrus clouds is unexpected, since on the large scale these clouds, unlike star-forming giant molecular clouds, are not gravitationally bound. Their turbulent kinetic energy is typically ten to one hundred times larger than their self-gravitational energy (Magnani et al. 1987; Heithausen 1996). As our observations show, even such cirrus clouds may contain substructures where gravity dominates, and which may be able to collapse to form stars.

Acknowledgements. We thank Peter Schilke for providing the LVG code used in this study. Many thanks to E. Kreysa and the MPIfR bolometer group for providing MAMBO, and to R. Zylka for the MOPSI data reduction package. This work was supported by the Deutsche Forschungsgemeinschaft grant SFB-494, and it made use of the IRAM 30 m telescope. IRAM is supported by INSU/CNRS (France), MPG (Germany), and IGN (Spain).

\section{References}

Bernard, J. P., Abergel, A., Ristorcelli, I., et al. 1999, A\&A, 347,640

Bertoldi, F., \& McKee, C. F. 1992, ApJ, 395, 140

Draine, B. T., \& Lee, H. M. 1984, ApJ, 285, 89

Falgarone, E., Panis, J. F., Heithausen, A., et al. 1998, A\&A, 331, 669

Feigelson, E. D. 1996, ApJ, 468, 306

Gerin, M, Falgarone, E., Joulain, K., et al. 1997, A\&A, 318, 579

Großmann, V., Heithausen, A., Meyerdierks, H., \& Mebold, U. 1990, A\&A, 240, 400

Großmann, V., \& Heithausen, A. 1992, A\&A, 264, 195

Gwenlan, C., Ruffle, D. P., Viti, S., Hartquist, T. W., \& Williams, D. A. 2000, A\&A, 354, 1127

Hearty, T., Magnani, L., Caillault, J. P., et al. 1999, A\&A, 341, 163

Hearty, T., Neuhäuser, R., Stelzer, B., et al. 2000, A\&A, 353, 104

Heithausen, A., \& Thaddeus, P. 1990, ApJ, 353, L49

Heithausen, A., Stacy, J. G., de Vries, H. W., Mebold, U., \& Thaddeus, P. 1993, A\&A, 268, 265 
Heithausen, A., Corneliussen, U., \& Großmann, V. 1995, A\&A, 301, 941

Heithausen, A. 1996, A\&A, 314, 251

Heithausen, A., Corneliussen, U., \& Großmann, V. 1999, in The physics and chemistry of the interstellar medium, ed. V. Ossenkopf, J. Stutzki, \& G. Winnewisser (CGAVerlag, Herdecke), 199

Heithausen, A. 1999, A\&A, 349, L53

Lafferty, W. J., \& Lovas, F. J. 1978, J. Phys. Chem. Ref. Data, 7,441

Magnani, L., Blitz, L., \& Mundy, L. 1987, ApJ, 295, 402

Mardones, D., Myers, P. C., Tafalla, M., Wilner, D. J., Bachiller, R., \& Garay, G. 1997, ApJ, 489, 719
Martin, E. L., \& Kun, M. 1996, A\&AS, 116, 467

Mezger, P. G., Wink, J. E, \& Zylka, R. 1990, A\&A, 228, 95

Motte, F., André, P., \& Neri, R. 1998, A\&A, 336, 150

Myers, P. C., Mardones, D., Tafalla, M., et al. 1996, ApJ, 465, L133

Nejad, L. A. M., \& Wagenblast, R. 1999, A\&A, 350, 204

Neuhäuser, R. 1999, Rev. Mod. Astr., 12, 27

Stutzki, J., \& Güsten, R. 1990, ApJ, 356, 513

Taylor, S. D., Morata, O., \& Williams, D. A. 1998, A\&A, 336, 309

Ward-Thompson, D., Motte, F., \& André, P. 1999, MNRAS, 305, 143 